\title{
Bemerkung zu dem Artikel Ein neuer elementarer Irrationalitätsbeweis für $\pi$ Math. Semesterber. 53:101-107 (2006)
}

\author{
Gisela Vetter
}

In dem gegebenen Irrationalitätsbeweis für $\pi$ sind zwei Fehler enthalten, auf die mich verschiedene Leser der Semesterberichte, zuerst Herr Dr. W. Fensch, Karlsruhe, und Herr Dr. E. Symeonides, Eichstätt-Ingolstadt, hingewiesen haben:

- Die Diskriminantenabschätzung auf S. 104 beruht auf falschen Werten und kann auch für andere $k \neq 20$ keine negativen Diskriminanten ergeben.

- Beim Übergang zur vorletzten Zeile auf S. 106 ist bei cos 1 ein Faktor 2 weggefallen.

Die Beweisführung ist somit falsch.

Eine Korrektur kann ich leider nicht vorlegen.

The online version of the original article can be found at http://dx.doi.org/10.1007/s00591-005-0103-z

G. Vetter

Doßstr. 9, 12621 Berlin, Germany 